\title{
ESTIMATIVA DA EVAPOTRANSPIRAÇÃO A PARTIR DE VARIAÇÃO DE NÍVEL ESTÁTICO DE AQUÍFERO ${ }^{1}$
}

\section{CARLOS E. A. G. BARRETO ${ }^{2}$, EDSON WENDLAND ${ }^{3}$, FRANCISCO F. N. MARCUZZO 4}

RESUMO: A quantificação da evapotranspiração é uma tarefa essencial para o gerenciamento dos recursos hídricos em uma bacia hidrográfica, já que é variável importante no ciclo hidrológico. Entretanto, estimar a evapotranspiração real é uma tarefa difícil, e os pesquisadores usam, geralmente, mais de um método a fim de validar os resultados. Este trabalho apresenta um método para a determinação da evapotranspiração real com base na variação da precipitação, do escoamento de base e do nível de água subterrânea medidas na bacia hidrográfica. Usando o método proposto em uma bacia hidrográfica piloto, a evapotranspiração real foi estimada em $900 \mathrm{~mm}$ por ano. Esse resultado foi comparado com a evapotranspiração potencial, calculada pelos métodos empíricos e semiempíricos, baseados em dados climatológicos. $\mathrm{O}$ método de Thornthwaite, com evapotranspiração potencial de $936 \mathrm{~mm}$ por ano, apresentou o valor mais próximo ao método de balanço hídrico. O método do balanço hídrico, baseado em medidas hidrogeológicas, mostrou-se apropriado para estimar a evapotranspiração real na bacia hidrográfica. Entretanto, é necessário observar as hipóteses e as limitações descritas neste estudo para aplicar este método a outra bacia hidrográfica.

PALAVRAS-CHAVE: gerenciamento de recursos hídricos, balanço hídrico, água subterrânea.

\section{ESTIMATING EVAPOTRANSPIRATION BASED ON GROUNDWATER LEVEL VARIATION IN A WATERSHED}

\begin{abstract}
Evapotranspiration quantification is a necessary task for water resources management in a watershed, since it is an important variable of the hydrological cycle. However, the estimation of the real evapotranspiration is a difficult task and researchers commonly use more than one method in order to validate the results. This paper presents a method for real evapotranspiration determination based on precipitation, runoff and groundwater level variation measured in the watershed. Using this method the real evapotranspiration in a pilot watershed was estimated to $900 \mathrm{~mm}$ per year. This result has been compared to potential evapotranspiration calculated by empirical and semi-empirical methods, based on climatologic data. The Thornthwaite method, with a potential evapotranspiration of $936 \mathrm{~mm}$ per year, presented the closest value to the water balance method. The water balance method based on hydrogeologic measurements showed to be suitable for real evapotranspiration estimation in the watershed. However it is necessary to observe the hypothesis and restrictions described in this paper to apply this method to another watershed.
\end{abstract}

KEYWORDS: water resources management, water balance, groundwater.

\footnotetext{
${ }^{1}$ Extraído da dissertação de mestrado do primeiro autor. Financiado pelo CNPq.

${ }^{2}$ Eng $^{\underline{0}}$ Civil, Doutorando em Engenharia de Petróleo, DEP-FEM-UNICAMP, Campinas - SP, agbarreto@ gmail.com

${ }^{3}$ Eng ${ }^{\mathrm{O}}$ Civil, Prof. Dr. Associado, SHS-EESC-USP, Caixa Postal 359, São Carlos - SP, ew@ @s.usp.br

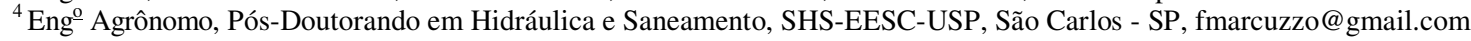
Recebido pelo Conselho Editorial em: 16-4-2008

Aprovado pelo Conselho Editorial em: 1ㅇ-4-2009
} 


\section{INTRODUÇÃO}

A Lei $\mathrm{n}^{\mathrm{o}}$ 9.433, de 1997, em seu artigo $7^{\circ}$, Inciso III, define que os Planos de Recursos Hídricos terão, como conteúdo mínimo, o balanço entre a disponibilidade e a demanda futura dos recursos hídricos em quantidade e qualidade, com identificação dos conflitos potenciais. Uma das principais parcelas que interferem na disponibilidade hídrica é a evapotranspiração, sendo necessário seu estudo para qualquer planejamento e/ou gestão de bacias hidrográficas.

Em algumas regiões, a quantidade de água evapotranspirada de uma bacia representa parcela superior à água que escoa superficialmente. Entretanto, a evapotranspiração é extremamente difícil de ser estimada com confiança, devido à inexistência de técnicas diretas que se apliquem a grandes áreas e à necessidade de instrumentos de custo elevado para a obtenção de estimativas indiretas. Além disso, "os resultados possíveis de serem obtidos têm de ser vistos com cautela por serem pontuais, tornando seu uso possível apenas por hipóteses simplificadoras ou longos períodos de tempo, em que pequenas variações se tornam desprezíveis" (MORTON, 1983).

As técnicas utilizadas para estimar a evapotranspiração em uma bacia hidrográfica baseiam-se no balanço hídrico, no uso do lisímetro ou na utilização de equações empíricas baseadas em dados climáticos. O fato é que as estimativas pelos diversos métodos, em geral, não apresentam os mesmos resultados, chegando, em alguns casos, a diferenças superiores a $100 \%$ entre dois métodos diferentes (BARRETO, 2006; MAZIERO, 2005). Verifica-se que é comum o uso de técnicas diferentes para a verificação das estimativas, visando a diminuir o nível de incerteza dos valores encontrados.

Alguns estudos que estimam a evapotranspiração têm sido desenvolvidos na bacia do Ribeirão da Onça e em áreas próximas ao Centro de Recursos Hídricos e Ecologia Aplicada da Universidade de São Paulo (CONTIN NETO, 1987; POMPÊO, 1990; MATTOS, 1991; ARANTES, 2003; CUNHA, 2003; BARRETO, 2006). Nessa região, encontram-se instalados equipamentos para medições diárias de dados climatológicos e de nível estático do aquífero.

Neste trabalho, a evapotranspiração real anual é estimada a partir da variação do nível do aquífero. O objetivo do trabalho é apresentar uma técnica que possa ser aplicada no auxílio do gerenciamento e do planejamento de uma bacia.

\section{MATERIAL E MÉTODOS}

\section{Bacia do Ribeirão da Onça}

A bacia do Ribeirão da Onça localiza-se na região do município de Brotas - SP, no centro-leste do Estado de São Paulo, entre os paralelos 22 $2^{\circ} 10^{\prime}$ e $22^{\circ} 15^{\prime}$ de latitude sul e entre os meridianos $47^{\circ} 55^{\prime}$ e $48^{\circ} 00^{\prime}$ de longitude oeste. O Ribeirão da Onça é um dos formadores do Rio Jacaré-Guaçu, afluente do Rio Tietê pela margem direita (Figura 1).

Uma característica importante dessa bacia é situar-se quase inteiramente em uma das regiões de afloramento do Sistema Aquífero Guarani (SAG), podendo fornecer elementos de interesse para o aprofundamento dos conhecimentos sobre o comportamento desse sistema, especialmente com relação à recarga profunda.

A temperatura média anual da região é de $20,5^{\circ} \mathrm{C}$, valor obtido na Estação Climatológica da área de estudo. A precipitação média anual varia entre 1.300 e $1.400 \mathrm{~mm}$. A classificação climática de Köeppen-Geiger para a região, classificada como $\mathrm{Cwa}$, indica que a bacia possui clima subtropical úmido com chuvas de verão, apresentando variação para clima tropical úmido com inverno seco. 


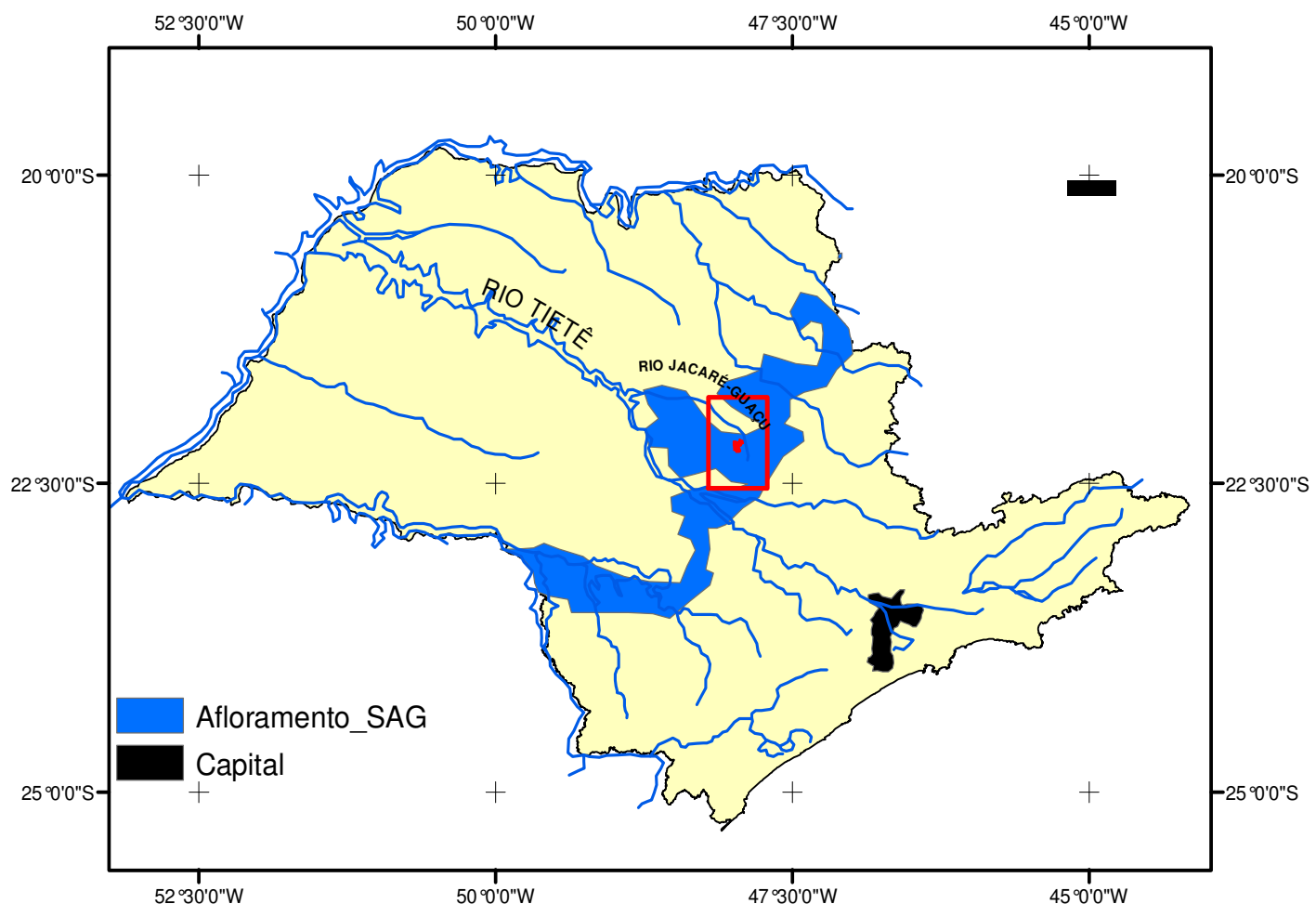

FIGURA 1. Localização geográfica da bacia do Ribeirão da Onça. Geographic location of the Ribeirão da Onça basin.

\section{Balanço hídrico}

O método empregado estima a evapotranspiração real (ETR - $\mathrm{mm} \mathrm{dia}^{-1}$ ) a partir de dados de precipitação $\left(\mathrm{P}-\mathrm{mm} \mathrm{dia}^{-1}\right)$, recarga direta $\left(\mathrm{R}_{\mathrm{d}}-\mathrm{mm} \mathrm{dia}^{-1}\right)$ e escoamento superficial direto (ES, run off $\left.-\mathrm{mm} \operatorname{dia}^{-1}\right)$.

$$
\mathrm{ETR}=\mathrm{P}-\mathrm{ES}-\mathrm{R}_{\mathrm{d}}
$$

A infiltração média na bacia corresponde à diferença entre precipitação $(\mathrm{P})$ e escoamento superficial direto (ES). Da parcela que infiltra, parte chega à zona saturada (recarga direta, $\mathrm{Rd}$ ) e parte fica na zona não saturada, podendo sair do sistema por evapotranspiração. Com o valor da recarga direta estimado a partir da variação do nível do aquífero, e assumindo a hipótese de que o balanço de umidade na zona não saturada é nulo no período anual, pode-se chegar a um valor para a evapotranspiração real anual na bacia.

O método para a estimativa da evapotranspiração real anual foi aplicado para regiões no entorno de poços de monitoramento. Em poços de monitoramento locados em terrenos relativamente planos, admitiu-se que o escoamento superficial direto seja igual a zero para a região no entorno do poço.

\section{Instrumentação e coleta de dados}

O método adotado neste trabalho está condicionado à existência de dados das principais variáveis do ciclo hidrológico local. As componentes relevantes adotadas do ciclo hidrológico foram: precipitação, evapotranspiração, escoamento superficial e recarga direta.

A bacia do Ribeirão da Onça conta com 23 poços de monitoramento, um pluviômetro, um linígrafo e uma régua linimétrica instalados dentro da bacia e em suas proximidades. As variáveis monitoradas na estação climatológica na área de estudo foram: insolação, velocidade do vento, evaporação, temperatura e umidade relativa do ar. 


\section{Dados climatológicos e precipitação}

$\mathrm{Na}$ Tabela 1, apresentam-se os dados climatológicos observados no Centro de Recursos Hídricos e Ecologia Aplicada da Universidade de São Paulo, no município de Itirapina, em 2005. Esses dados foram utilizados na estimativa da ETP pelos diferentes métodos utilizados. A precipitação anual observada durante o período de estudo foi de $1.424,8 \mathrm{~mm}$. Observa-se que $38 \%$ da precipitação anual se concentrou no mês de janeiro. No período seco, de abril a setembro, as precipitações representaram apenas $11 \%$ do total anual.

TABELA 1. Dados coletados na Estação Climatológica do CRHEA-USP, Itirapina - SP. Data collected at the Climatologic Station CRHEA-USP, Itirapina - SP, Brazil.

\begin{tabular}{|c|c|c|c|c|c|c|c|c|c|}
\hline \multirow[b]{2}{*}{ Tempo } & \multicolumn{3}{|c|}{ Temperatura } & \multirow{2}{*}{$\begin{array}{l}\text { Umidade } \\
\text { Relativa }\end{array}$} & \multirow{2}{*}{$\begin{array}{c}\text { Evaporação } \\
\text { Tanque } \\
\text { Classe "A" }\end{array}$} & \multirow[b]{2}{*}{ Precipitação } & \multirow{2}{*}{$\begin{array}{l}\text { Vento } \\
(2 \mathrm{~m})\end{array}$} & \multirow{2}{*}{$\begin{array}{l}\text { Luz } \\
\text { solar }\end{array}$} & \multirow[b]{2}{*}{ Insolação } \\
\hline & máx. & mín. & méd. & & & & & & \\
\hline Meses & \multicolumn{3}{|c|}{ - } & $\%$ & ------------ & Im ----------- & $\mathrm{m} \mathrm{s}^{-1}$ & $(\%)$ & horas \\
\hline jan. & 32,0 & 13,4 & 23,1 & 88,0 & 171,9 & 542,3 & 1,0 & 0,25 & 4,1 \\
\hline fev. & 34,4 & 10,5 & 23,3 & 80,3 & 152,1 & 69,0 & 0,9 & 0,26 & 8,9 \\
\hline mar. & 33,3 & 11,9 & 23,7 & 83,5 & 149,8 & 155,1 & 0,7 & 0,27 & 6,8 \\
\hline abr. & 32,8 & 9,8 & 22,9 & 79,7 & 117,7 & 13,1 & 0,6 & 0,28 & 7,2 \\
\hline maio & 30,2 & 6,0 & 18,7 & 71,2 & 127,9 & 87,9 & 0,5 & 0,29 & 7,5 \\
\hline jun. & 27,4 & 8,3 & 18,0 & 88,2 & 94,0 & 41,5 & 0,7 & 0,30 & 7,6 \\
\hline jul. & 28,8 & 7,0 & 16,1 & 84,9 & 112,9 & 4,3 & 1,1 & 0,30 & 7,8 \\
\hline ago. & 33,2 & 6,0 & 19,3 & 75,6 & 170,0 & 16,1 & 0,8 & 0,29 & 9,2 \\
\hline set. & 31,6 & 4,6 & 19,8 & 86,9 & 141,3 & 85,0 & 1,9 & 0,28 & 6,8 \\
\hline out. & 34,8 & 12,6 & 24,0 & 87,0 & 152,8 & 84,8 & 1,3 & 0,26 & 6,0 \\
\hline nov. & 33,4 & 10,5 & 24,1 & 86,2 & 173,6 & 100,7 & 1,6 & 0,25 & 6,7 \\
\hline dez. & 32,2 & 10,6 & 23,1 & 78,0 & 181,9 & 225,0 & 1,5 & 0,25 & 6,1 \\
\hline Média & 32,0 & 9,3 & 21,3 & 82,5 & 145,5 & 118,7 & 1,1 & 0,27 & 7,1 \\
\hline
\end{tabular}

\section{Estimativa do escoamento superficial}

Para a estimativa do escoamento superficial direto anual, utilizou-se do modelo IPHS1 (TUCCI et al., 1989). Para a simulação hidrológica da bacia, o modelo utilizado é baseado no método da curva número (CN) desenvolvido pelo Soil Conservation Service (SCS, 1972). A principal vantagem desse método é a existência de apenas um parâmetro, o $\mathrm{CN}$, o qual deve ser calibrado para a área estudada. A calibração foi realizada com dados de um linigrama, instalado em uma seção do Ribeirão da Onça. Executou-se a calibração pelo parâmetro de volume escoado, sem verificação do tempo de pico nem do tempo de recessão do hidrograma simulado (TUCCI et al., 1989).

A calibração do modelo chuva-vazão foi realizada em três situações: uma para condições de baixa umidade do solo e duas para condições de alta umidade do solo. A condição para o solo ser considerado de baixa umidade foi a não ocorrência de precipitação total maior do que $36 \mathrm{~mm}$ nos últimos 5 dias e em período menor não terem ocorrido precipitações que, somadas, totalizem $13 \mathrm{~mm}$. Em qualquer outra condição, o solo é considerado de alta umidade.

Para o período seco, calibrou-se o hidrograma em dois dias consecutivos com chuva (25 e 26-11-2005). Para a calibração do período úmido, foram usadas as precipitações ocorridas nos dias $1^{\mathrm{o}}$; 2; 3-12-2005, para a primeira simulação, e as precipitações ocorridas nos dias 5; 6 e 7-12-2005, para a segunda.

\section{Determinação da recarga direta}

O Método da Flutuação da Superfície Livre (WTF - Water Table Fluctuation) é a técnica mais empregada para a estimativa da recarga. O método WTF baseia-se na premissa de que as elevações 
nos níveis d'água em aquíferos não confinados são devidas à água de recarga subterrânea chegando à superfície livre. A recarga é, durante um intervalo de tempo predefinido, calculada pela eq. (2) (HEALY \& COOK, 2002; SCANLON et al., 2002; MOON et al., 2004):

$$
\operatorname{Rd}=S_{y} \frac{\Delta h}{\Delta t}
$$

em que,

$\mathrm{Rd}$ - estimativa de recarga direta, $\mathrm{m} \mathrm{dia}^{-1}$;

$\mathrm{S}_{\mathrm{y}}$ - rendimento específico, adimensional;

$\mathrm{h}$ - altura do nível freático, $\mathrm{m}$, e

$\mathrm{t}$ - tempo, dias.

O rendimento específico de um aquífero livre é, em muitos casos, igual ao coeficiente de armazenamento, sendo da ordem da porosidade útil do meio.

A eq. (2) assume que a água que atinge o nível freático entra imediatamente em armazenamento e que todos os outros componentes do balanço hídrico subterrâneo (evapotranspiração subterrânea, fluxo de base, entrada e saída de fluxo subsuperficial) são nulos durante o período de recarga.

\section{Estimativa da evapotranspiração através de métodos empíricos}

A evapotranspiração potencial na bacia foi estimada por meio de métodos empíricos e semiempíricos. Dados necessários para os modelos foram coletados na estação climatológica da área de estudo.

Os métodos utilizados para estimar a evapotranspiração foram: Penman-Monteith, Thornthwaite, Tanque Classe A (FAO), Blaney-Criddle e Makkink.

Para o cálculo da evapotranspiração real na bacia, foi utilizada a eq. (3):

$$
\mathrm{ET}_{\text {est }}=\sum_{\mathrm{i}=1}^{\mathrm{n}}\left(\mathrm{kc}_{\mathrm{i}} \text { ETP } \mathrm{Ar}_{\mathrm{i}}\right)
$$

em que,

$\mathrm{ET}_{\mathrm{est}}$ - evapotranspiração real da bacia, mm;

$\mathrm{k}_{\mathrm{ci}}$ - coeficiente de cultura médio para a cultura i (adimensional) em todo o período de cultivo;

ETP - evapotranspiração, mm, e

$A r_{i}$ - área relativa da cultura i em relação à área total da sub-bacia, adimensional.

Os coeficientes $\mathrm{k}_{\mathrm{c}}$ variam para a mesma cultura, dependendo da fase de desenvolvimento. $\mathrm{Na}$ Tabela 2, estão apresentados os valores de kc para cada fase de desenvolvimento das principais culturas e tipos de vegetação da bacia. Adotaram-se valores médios de $\mathrm{kC}$, sendo obtidos da média ponderada relativa ao período de duração de cada etapa de desenvolvimento da cultura/vegetação.

Para o cálculo de $A r_{i}$, é necessário determinar as áreas de cada cultura dentro da sub-bacia do Ribeirão da Onça, que fica a montante do posto fluviométrico. A determinação foi feita a partir de imagens de satélite e de verificação em campo. A bacia do Ribeirão da Onça é de uso agrário e pecuário, não sendo observadas áreas pavimentadas ou industriais. 
TABELA 2. Distribuição das culturas na bacia em percentagem e coeficientes $\mathrm{k}_{\mathrm{c}}$ recomendados pela FAO (ALLEN et al., 1998). Distribution of the cultures in the basin in percentage and kc coefficients recommended by FAO (ALLEN et al., 1998).

\begin{tabular}{|c|c|c|c|c|c|c|c|}
\hline \multirow{2}{*}{ Cultura } & \multirow{2}{*}{$\begin{array}{c}\text { Área } \\
\%\end{array}$} & & \multicolumn{4}{|c|}{ Etapa de Desenvolvimento } & \multirow{2}{*}{$\begin{array}{c}\text { kc } \\
\text { Médio }\end{array}$} \\
\hline & & & $\mathrm{I}$ & II & III & IV & \\
\hline \multirow{2}{*}{ Mata Ciliar } & \multirow{2}{*}{12} & duração ${ }^{1}$ & - & - & - & - & \multirow{2}{*}{1,00} \\
\hline & & $\mathrm{kc}$ & 1 & 1 & 1 & 1 & \\
\hline \multirow{2}{*}{ Cerrado } & \multirow{2}{*}{2} & duração & - & - & - & - & \multirow{2}{*}{1,00} \\
\hline & & $\mathrm{kc}$ & 1 & 1 & 1 & 1 & \\
\hline \multirow{2}{*}{ Eucalipto } & \multirow{2}{*}{41} & duração & - & - & - & - & \multirow{2}{*}{1,00} \\
\hline & & $\mathrm{kc}$ & 1 & 1 & 1 & 1 & \\
\hline \multirow{2}{*}{ Citros } & \multirow{2}{*}{21} & duração & 150 & 90 & 90 & 35 & \multirow{2}{*}{0,80} \\
\hline & & $\mathrm{kc}$ & 0,8 & 0,8 & 0,8 & 0,8 & \\
\hline \multirow{2}{*}{ Cana-de-açúcar } & \multirow{2}{*}{9} & duração & 8 & 15 & 45 & 24 & \multirow{2}{*}{1,04} \\
\hline & & $\mathrm{kc}$ & 0,4 & 0,825 & 1,25 & 1 & \\
\hline \multirow{2}{*}{ Pasto } & \multirow{2}{*}{8} & duração & 140 & 60 & 120 & 45 & \multirow{2}{*}{0,90} \\
\hline & & $\mathrm{kc}$ & 0,85 & 0,9 & 0,95 & 0,9 & \\
\hline \multirow{2}{*}{ Soja } & \multirow{2}{*}{7} & duração & 20 & 30 & 60 & 25 & \multirow{2}{*}{0,90} \\
\hline & & $\mathrm{kc}$ & 0,4 & 0,775 & 1,15 & 0,875 & \\
\hline
\end{tabular}

${ }^{1}$ Duração em dias.

\section{RESULTADOS E DISCUSSÃO}

\section{Escoamento superficial direto}

O volume total de escoamento direto estimado pelo modelo chuva-vazão foi de $5.415 .552 \mathrm{~m}^{3}$. $\mathrm{O}$ volume escoado transformado em altura equivalente de água sobre a área da bacia foi de $102,28 \mathrm{~mm}$ (Tabela 3). Esse resultado corresponde a 7\% da precipitação anual. CONTIN NETO (1987) apresenta resultado para o escoamento direto da bacia do Ribeirão da Onça na ordem de 1,5\% da precipitação anual, tendo utilizado o método gráfico de separação do escoamento de base e do escoamento superficial. Porém, o valor precipitado no período estudado por CONTIN NETO (1987) foi da ordem de $1.000 \mathrm{~mm}$, abaixo da média histórica.

O mês de janeiro apresentou cerca de $68 \%$ do escoamento direto anual. A média de chuva a longo prazo para o mês de janeiro nessa região está em torno de $240 \mathrm{~mm}$. Em 2005, a precipitação de janeiro foi de $542,3 \mathrm{~mm}$, mais do que o dobro da média histórica, afetando diretamente o escoamento superficial direto. Por outro lado, no mês de fevereiro, a precipitação foi de $69,0 \mathrm{~mm}$, que está abaixo da média de longo termo de $200 \mathrm{~mm}$, o que ocasionou um escoamento superficial direto muito baixo.

TABELA 3. Escoamento superficial mensal simulado pelo método de transformação chuva-vazão para o ano de 2005, em altura equivalente de água sobre a área da bacia. Monthly superficial drainage simulated by the rain-discharge model for the year 2005 , in equivalent water column for the basin.

\begin{tabular}{ccccccccccccc}
\hline Mês & jan. & fev. & mar. & abr. & maio & jun. & jul. & ago. & set. & out. & nov. & dez. \\
\hline $\begin{array}{c}\text { Escoamento } \\
\begin{array}{c}\text { Superficial } \\
(\mathrm{mm})\end{array}\end{array}$ & 68,63 & 0,54 & 16,00 & 0,00 & 7,57 & 0,00 & 0,00 & 0,00 & 0,31 & 0,00 & 3,20 & 6,51 \\
\hline
\end{tabular}

\section{Avaliação da recarga direta}

O valor anual da recarga direta foi obtido pela média aritmética da recarga direta obtida em cada poço de observação. Para uma porosidade útil considerada de 0,10 a 0,13 no período de 1-1-2005 a 31-12-2005, a recarga média foi de 313 a $407 \mathrm{~mm}$, respectivamente. Na Tabela 4, apresentam-se os valores individuais para os poços analisados. 
TABELA 4. Recarga direta (mm) estimada no entorno dos poços em 2005. Estimated direct recharge ( $\mathbf{m m})$ for the wells in 2005.

\begin{tabular}{ccccc}
\hline Poço & Cultura no Entorno & $\begin{array}{c}\text { Profundidade Média do } \\
\text { Nível no Poço }(\mathrm{m})\end{array}$ & $\mathrm{S}_{\mathrm{y}}=10 \%$ & $\mathrm{~S}_{\mathrm{y}}=13 \%$ \\
\hline 3 & Pasto & 16,05 & 215 & 278 \\
5 & Pasto & 5,54 & 228 & 296 \\
8 & Laranja & 21,64 & 534 & 694 \\
9 & Eucalipto & 20,54 & 37 & 48 \\
13 & Laranja & 9,24 & 324 & 421 \\
15 & Cana-de-açúcar & 6,94 & 258 & 335 \\
16 & Pasto & 4,58 & 145 & 188 \\
18 & Pasto & 12,51 & 541 & 603 \\
19 & Pasto & 13,57 & 536 & 697 \\
\hline
\end{tabular}

O valor estimado para a evapotranspiração por meio da recarga direta, da precipitação e do escoamento superficial foi de $900 \mathrm{~mm}$, para a porosidade útil de $13 \%$, e de $994 \mathrm{~mm}$, para a porosidade de $10 \%$. Comparando-se esses valores da evapotranspiração real com a estimativa da evapotranspiração real na bacia estimada pelo método de Thornthwaite, que foi de $936 \mathrm{~mm}$, temos diferença de $3 \%$ e $-6 \%$. De acordo com essa análise, a evapotranspiração da bacia representa, em média, $67 \%$ da precipitação.

\section{Métodos empíricos para cálculo da evapotranspiração real}

Os dados climatológicos apresentados anteriormente na Tabela 2 foram utilizados para o cálculo da evapotranspiração mensal, usando os métodos teóricos de estimativa. Na Figura 2, apresentam-se as estimativas da evapotranspiração potencial mensal para a região do Ribeirão da Onça.

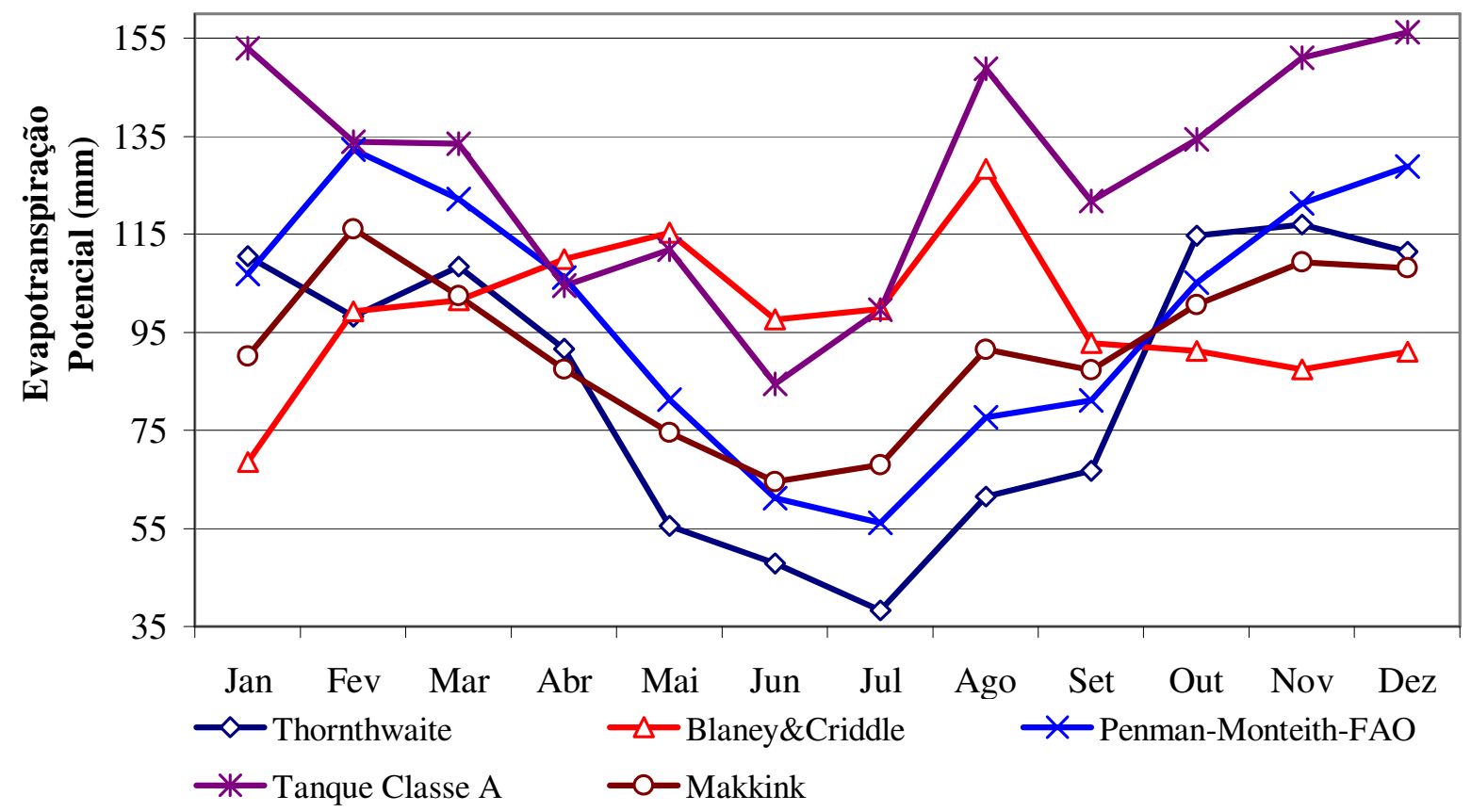

FIGURA 2. Evapotranspiração potencial mensal estimada para o ano de 2005 por meio de diferentes métodos. Monthly estimated potential evapotranspiration by different methods for the year 2005. 
A evapotranspiração real anual na bacia do Ribeirão da Onça, corrigida de acordo com a área relativa das diferentes culturas, é apresentada na Tabela 5.

TABELA 5. Evapotranspiração real anual na bacia do Ribeirão da Onça, em 2005, estimada por meio de diferentes métodos. Annual actual evapotranspiration in the Ribeirão da Onça basin in 2005, estimated by different methods.

\begin{tabular}{lc}
\hline Método & Evapotranspiração na Sub-bacia $(\mathrm{mm})$ \\
\hline Penman-Monteith (FAO) & 1.082 \\
Thornthwaite & 936 \\
Tanque Classe A (FAO) & 1.399 \\
Blaney-Criddle & 1.079 \\
Makkink & 1.005 \\
\hline
\end{tabular}

O método de Thornthwaite, que indicou uma $\mathrm{ETR}_{\mathrm{T}}$ de $936 \mathrm{~mm}$, vem sendo apontado como o melhor método para a estimativa da evapotranspiração para a região (MATOS, 1987; CUNHA, 2003).

O método de Penman-Monteith é apontado pela FAO como o método que melhor representa as condições reais de evapotranspiração. Seus resultados neste estudo $\left(\mathrm{ETR}_{\mathrm{PM}}=1.082 \mathrm{~mm}\right)$ não diferiram muito dos encontrados pelo método de Thornthwaite, apesar de apresentarem-se na maior parte dos meses com um valor maior, sendo a correlação entre os dois métodos de 0,92. Esse comportamento semelhante pode indicar que as componentes da radiação solar e da temperatura são os fatores determinantes para a estimativa da evapotranspiração da região.

$\mathrm{O}$ método de Makkink apresentou resultado anual $\left(\mathrm{ETR}_{\mathrm{M}}=1.005 \mathrm{~mm}\right)$ equivalente à média dos métodos de Thornthwaite e Penman-Monteith da FAO. Isso já poderia ser esperado, pois o método de Makkink é derivado do método de Penman-Monteith. O coeficiente de correlação entre os métodos de Penman-Monteith e Makkink foi de 0,92, indicando que esse método é boa alternativa para a região.

O método de Blaney-Criddle apresentou resultado anual mais divergente. Observou-se crescimento na evapotranspiração no período de janeiro a maio e queda na evapotranspiração a partir de agosto, enquanto os outros métodos indicaram variação inversa. Isso pode ser explicado pelo fato de o método ter sido desenvolvido para regiões áridas e semiáridas, onde o período seco é também o período com temperatura mais elevada. No entanto, o total anual $\left(E_{T} R_{B C}=1.079 \mathrm{~mm}\right)$ é compatível com a estimativa pelo método de Penman-Monteith da FAO.

Pode-se afirmar, então, que a estimativa pelo método de Thornthwaite é o que mais se aproxima da evapotranspiração real na bacia. Essa conclusão foi apresentada por CUNHA (2003), a partir de estudos com lisímetro na mesma região.

Na Tabela 6, apresenta-se o resumo dos valores do ciclo hidrológico usados no método.

TABELA 6. Resumo dos valores das componentes do ciclo hidrológico usados no método. Summary of hydrologic cycle values used in the method.

\begin{tabular}{lcc}
\hline & Método 3 & \\
\hline \multirow{2}{*}{ Componente do Ciclo } & $\mathrm{S}_{\mathrm{y}}=10 \%$ & $\mathrm{~S}_{\mathrm{y}}=13 \%$ \\
\hline Precipitação & 1.410 & 1.410 \\
Escoamento superficial & 103 & 103 \\
Recarga direta & 313 & 407 \\
Evapotranspiração real & 994 & 900 \\
\hline
\end{tabular}


Verifica-se, pela Tabela 6, que o escoamento superficial é da ordem de $7 \%$ da precipitação anual, a recarga direta está na faixa de $22 \%$ a $29 \%$ e a evapotranspiração real anual está na faixa de $64 \%$ a $71 \%$. A incerteza nesses valores está relacionada diretamente ao valor da porosidade útil, já que a sensibilidade no cálculo da recarga direta é alta para esse parâmetro. Nesse modelo, não foram consideradas as incertezas na estimativa da precipitação e do escoamento superficial.

Os resultados podem ser utilizados para a averiguação de métodos de estimativa de evapotranspiração e como dado de entrada em modelos de representação do balanço hídrico em bacias. Os resultados deste trabalho mostram que esta técnica pode ser viável em locais específicos, onde as hipóteses e as restrições do método de estimativa da recarga do aquífero e relativas ao balanço de umidade da zona não saturada do solo possam ser aceitas.

\section{CONCLUSÕES}

Os valores de evapotranspiração potencial máxima, estimados pelo método de Thornthwaite, são os que mais se aproximam da evapotranspiração real na bacia estimada pelo balanço hídrico, utilizando dados do nível do aquífero. Para a verificação da eficácia deste método, é necessário um estudo mais prolongado. O método de estimativa da evapotranspiração anual por meio de nível de aquífero apresentou valores aproximados aos encontrados por meio do método de Thornthwaite, amplamente apontado em trabalhos anteriores como o método que melhor representa a evapotranspiração na região do estudo. Portanto, conclui-se que o método pode ser aplicado, desde que sejam observadas suas restrições.

\section{AGRADECIMENTOS}

Ao CNPq (141159/2006-5), à SG/OEA e à FAPESP, pelo fomento.

\section{REFERÊNCIAS}

ALLEN, R.G.; PEREIRA, L.S.; RAES, D.; SMUTH, M. Crop evapotranpiration: guidelines for computing crop water requirements. Rome: FAO, 1998301 p. (Irrigation and Drainage, Paper 56.)

ARANTES, E.J. Emprego de infiltrômetros na caracterização da interação entre rio e aquífero. 2003. 88 f. Dissertação (Mestrado em Hidráulica e Saneamento) - Escola de Engenharia de São Carlos, Universidade de São Paulo, São Carlos, 2003.

BARRETO, C.E.A.G. Balanço hídrico em zona de afloramento do Sistema Aquífero Guarani a partir de monitoramento hidrogeológico em bacia representativa. 2006. 149 f. Dissertação (Mestrado em Hidráulica e Saneamento) - Escola de Engenharia de São Carlos, Universidade de São Paulo, São Carlos, 2006.

CONTIN NETO, D. Balanço hídrico em bacia hidrográfica situada em região de recarga do Aquífero Botucatu. 1987. 182 f. Tese (Doutorado em Hidráulica e Saneamento) - Escola de Engenharia de São Carlos, Universidade de São Paulo, São Carlos, 1987.

CUNHA, A.T. Estimativa experimental da taxa de recarga na zona de afloramento do Aquífero Guarani, para a região de São Paulo - SP. 2003. 117 f. Dissertação (Mestrado em Hidráulica e Saneamento) - Escola de Engenharia de São Carlos, Universidade de São Paulo, São Carlos, 2003.

HEALY, R.W.; COOK, P.G. Using groundwater levels to estimate recharge. Hydrogeology Journal, New York, v.10, n.1, p. 91-109, 2002.

MATOS, I.S. Aplicação de um modelo de balanço hídrico na bacia do Rio Jacaré-Guaçu. 1987. 132 f. Dissertação (Mestrado em Hidráulica e Saneamento) - Escola de Engenharia de São Carlos, Universidade de São Paulo, São Carlos, 1987.

MATTOS, A. Evapotranspiração real: como determiná-la em estudos hidrológicos. In: SIMPÓSIO LUSO-BRASILEIRO DE HIDRÁULICA E RECURSOS HÍDRICOS, 5., SIMPÓSIO 
BRASILEIRO DE RECURSOS HÍDRICOS, 9., 1991, Rio de Janeiro. Anais... Porto Alegre: Associação Brasileira de Recursos Hídricos, 1991. p.168-177.

MAZIERO, T.A. Monitoramento de água subterrânea em área urbana: aspectos quantitativos. 2005. 85 f. Dissertação (Mestrado em Hidráulica e Saneamento) - Escola de Engenharia de São Carlos, Universidade de São Paulo, São Carlos, 2005.

MOON, S.; WOO, N.C.; LEE, K.S. Statistical analysis of hydrographs and water-table fluctuation to estimate groundwater recharge. Journal of Hydrology, New York, v.292, n.1, p.198-209, 2004.

MORTON, F.I. Operational estimates of areal evapotranspiration and their significance to the science and practice of hydrology. Journal of Hydrology, New York, v.66, n.1, p.1 - 76, 1983.

POMPÊO, C.A. Balanço hídrico da zona não saturada do solo na Bacia do Ribeirão do Onça - SP. 1990. 175 f. Tese (Doutorado em Hidráulica e Saneamento) - Escola de Engenharia de São Carlos, Universidade de São Paulo, São Carlos, 1990.

SCANLON, B.R.; HEALY, R.W.; COOK, P.G. Choosing appropriate techniques for quantifying groundwater recharge. Hydrogeology Journal, New York, v.10, n. 1, p. 18-39, 2002.

SCS. SOIL CONSERVATION SERVICE. Design Hydrographs. In: National Engineering Handbook. Washington: USDA, 1972. 127 p.

TUCCI, C.E.M.; ZAMANILLO, E.A.; PASINATO, H.D. Sistema de simulação precipitação-vazão IPHS1. Porto Alegre: IPH/UFRGS, 1989. 66 p. 